\title{
Le nom « Saguenay » dans l’histoire ${ }^{1}$
}

\author{
Mgr Victor Tremblay
}

\section{Introduction}

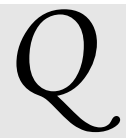

uatre entités géographiques portent actuellement le nom Saguenay: une région, un cours d'eau, une division électorale, un canton. La région (toujours la même), de temps immémorial; le cours d'eau (fjord et rivière), depuis 1535; la division électorale (d'étendue et de localisation variables), depuis 1829; le canton, depuis 1863.

Pour éviter toute confusion, dans certains contextes pouvant prêter à équivoque, on emploie l'expression « Royaume du Saguenay » lorsqu'il s’agit de la région, " rivière Saguenay " ou « fjord Saguenay », selon le cas, quand il s'agit du cours d'eau; « comté de

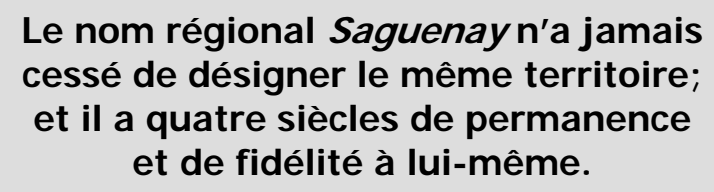
cessé de désigner le même territoire; et il a quatre siècles de permanence et de fidélité à lui-même.

les conditions d'authenticité. Labrador vient de l'étranger, du portugais, et ne cadre pas par le sens du mot, qui signifie "laboureur», avec le pays qu'il désigne; Canada a changé plus d'une fois d'application au cours de l'histoire, il a désigné tantôt un petit pays sauvage caractérisé par des groupes de cabanes, tantôt l'immense colonie française, tantôt une province, avant de s'appliquer à notre grand pays actuel, « a mari usque ad mare »; Hochelaga s'applique aujourd'hui à un quartier de ville, chose différente de la bourgade disparue qui portait originairement ce nom. Tandis que le nom régional Saguenay n’a jamais cessé de désigner le même territoire; et il a quatre siècles de permanence et de fidélité à lui-même.

Ce territoire a été indiqué Saguenay », pour désigner la division électorale; dans le cas du canton, il faut toujours mettre ce mot devant le nom Saguenay.

\section{Saguenay nom de région}

\section{Son origine et sa pérennité}

\section{L'origine du nom}

Par son origine le nom de la région du Saguenay se place en tête des plus anciens et des plus authentiques qu'on puisse trouver dans la toponymie canadienne.

Ce nom, qui signifie « eau qui sort », «d'où l'eau sort ", désignait un pays particulier de la vallée du Saint-Laurent avant la venue des blancs; il désignait ce pays par un trait descriptif qui le caractérise: le pays d'où viennent les eaux qui vont, d'un côté, au grand fleuve et, de l'autre, à la baie James; et depuis ce temps ancien il n'a pas cessé de s'appliquer au même territoire. Certains autres noms, comme Labrador, Canada, Hochelaga, sont aussi anciens que Saguenay, mais ils ne remplissent pas comme lui toutes avec précision au découvreur du Canada, Jacques Cartier, par les habitants du pays, les Indiens. Il s'étendait depuis la rive du Saint-Laurent, entre l'embouchure de la rivière Moisie (en bas de Sept-Iles) et l'île d'Orléans, jusqu'à une distance de "une lune » de trajet vers l'ouest à partir de Tadoussac, ce qui reporte à une centaine de milles à l'ouest du lac Saint-Jean. Il n'y a donc aucune équivoque possible sur l'identité du territoire primitivement appelé Saguenay et celui que nous continuons d'appeler ainsi et qui contient les six régions de la Côte-Nord, de Chicoutimi, du Lac SaintJean, de Chibougamau, de Charlevoix et du Parc des Laurentides. .

\section{La pérennité du nom}

La désignation de cette région par le nom « Saguenay » et l'identité du territoire désigné par ce nom s'appuient sur une tradition historique dont il est facile de démontrer la continuité par une suite de témoignages depuis l'origine jusqu'à maintenant. Citer tous ces témoignages serait trop long, fastidieux et inutile; contentons-nous de mentionner les plus connus qui font autorité. 
Jacques Cartier, le premier qui en a révélé l’existence et a inscrit le Saguenay dans la géographie et l'histoire, en 1535.

Le sieur de Roberval, qui à titre de vice-roi a tenté de s’y rendre en 1543.

Le géographe Jean Alfonse, qui est venu étudier les lieux, en a fait une description dans sa Cosmographie universelle et a été le premier à représenter le lac Saint-Jean, qu’il a appelé « la mer du Saguenay », en 1543.

Les neveux et arrière-neveux de Cartier, leurs associés et leurs concurrents, qui ont fréquenté les lieux pendant 45 ans.

Les rois de France François $1^{\mathrm{er}}$, Henri III, Henri IV, Louis XIII, qui ont inscrit le pays du Saguenay dans les documents officiels, et Louis XIV, qui en a fait, en 1674 , un "domaine du roi », transmis à ses successeurs et ensuite aux rois d'Angleterre.

Samuel de Champlain, qui a plus d'une fois voulu compléter l'exploration du pays du Saguenay en parcourant le circuit indiqué par Cartier.

Les habitants du Saguenay: Mistassins, Nekoubauistes, Piékouagamiens, Chicoutimiens, Tadoussaciens, Betsiamites, Papinachois, Oumanieks.

Les commerçants de fourrures, qui ont fréquenté le pays du Saguenay pendant 300 ans, et les missionnaires, qui l'ont parcouru en tous sens pendant 140 ans.

Les cartes géographiques anciennes, qui, au nombre de plus de 70 dans la période de 300 ans qui a précédé la colonisation, inscrivent le nom SAGUENAY comme désignation d'un pays ${ }^{2}$.

L’ingénieur Joseph-Laurent Normandin, qui est venu marquer les limites du territoire du Saguenay à titre de « domaine du roi » en 1732.

Une équipe de légistes et d'archivistes a, de nos jours (en 1926), démarqué par une ligne le contour de ce Domaine du Roi sur la carte dressée par eux pour baser le jugement du Conseil Privé d'Angleterre au sujet des limites entre le Labrador et la province de Québec. (Nous donnons plus loin une reproduction de la partie de cette carte qui représente la région du Saguenay).
Paschal Taché, père et fils, qui ont passé une grande partie de leur carrière dans le Saguenay et en ont dessiné une carte en 1725.

Marc-Paschal de Sales Laterrière, député du Saguenay pendant 17 ans, le notaire Ch.-H. Gauvreau, le grand chef indien Nicolas Vincent, consultés par l'Assemblée législative comme particulièrement connnaissants sur le territoire du Saguenay, en 1827 et 1828.

Les députés du Bas-Canada, qui, en 1828, créaient une " commission pour explorer le Saguenay » en vue de la colonisation.

Les « commissaires explorateurs du territoire du Saguenay », les arpenteurs, géologues et ingénieurs qui ont exécuté cette exploration de la région en tous sens, dans l'été de 1828.

Les députés qui, en 1829, donnaient le nom de «Comté de Saguenay » au district électoral correspondant à cette région.

Les membres de la Société des Vingt-et-Un, qui ont ouvert le Saguenay à la colonisation, en 1838.

Ceux de la Société des Défricheurs de la Rivière-auSable, qui ont colonisé le canton Jonquière, en 1847.

Ceux de l'Association des comtés de l'Islet et de Kamouraska pour coloniser le Saguenay, qui ont commencé la colonisation de la région du Lac SaintJean, à Hébertville, en 1849.

Les arpenteurs D.-S. Ballantyne, P.-A. Tremblay, Louis Legendre, J.-B. Duberger, P.-H. Dumais et autres spécialistes de la précision géographique, qui ont tous reconnu sous le nom de Saguenay le même territoire.

Mgr Dominique Racine, l'éminent connaisseur et réalisateur, qui, curé pendant 16 ans et premier évêque de Chicoutimi, a mérité le titre d' "Apôtre du Saguenay ", et après lui tous ses successeurs, qui ont comme lui associé le nom de la grande région du Saguenay à celui de leur diocèse.

Les Délégués Apostoliques, qui, du premier au dernier, ont fait visite au « Royaume du Saguenay ».

Les fondateurs de nos journaux régionaux (Le Saguenay, Le Réveil du Saguenay, Le Progrès du Saguenay, 
Le Lac-Saint-Jean, Le Rapatriement, Le Colon, L’Étoile du Lac, Le Lingot...); maints auteurs (Drapeau, Huard, Béchard, Chambers, Murray, Provancher, Potvin, Barbeau, etc.); nos députés les plus remarquables (Étienne Parent, David et William Price, P.-A. Tremblay, Ernest Cimon, Élie St-Hilaire, Joseph Girard, Vilmont et Edmond Savard, Émile Moreau... pour ne nommer que des disparus); plus d'un ancien maire d'Hébertville, de Roberval, de Saint-Jérôme, SaintPrime, de Saint-Félicien, de Chicoutimi, etc.; nombre de curés de toutes les parties du diocèse, et combien d'autres! Nous avons relevé plus de mille citations de l'emploi du nom du Saguenay comme désignation de la région.

\section{Carte du territoire du Saguenay tel que réservé comme Domaine du Roi en 1674. Contour tracé en 1926 par le Conseil privé d'Angleterre}

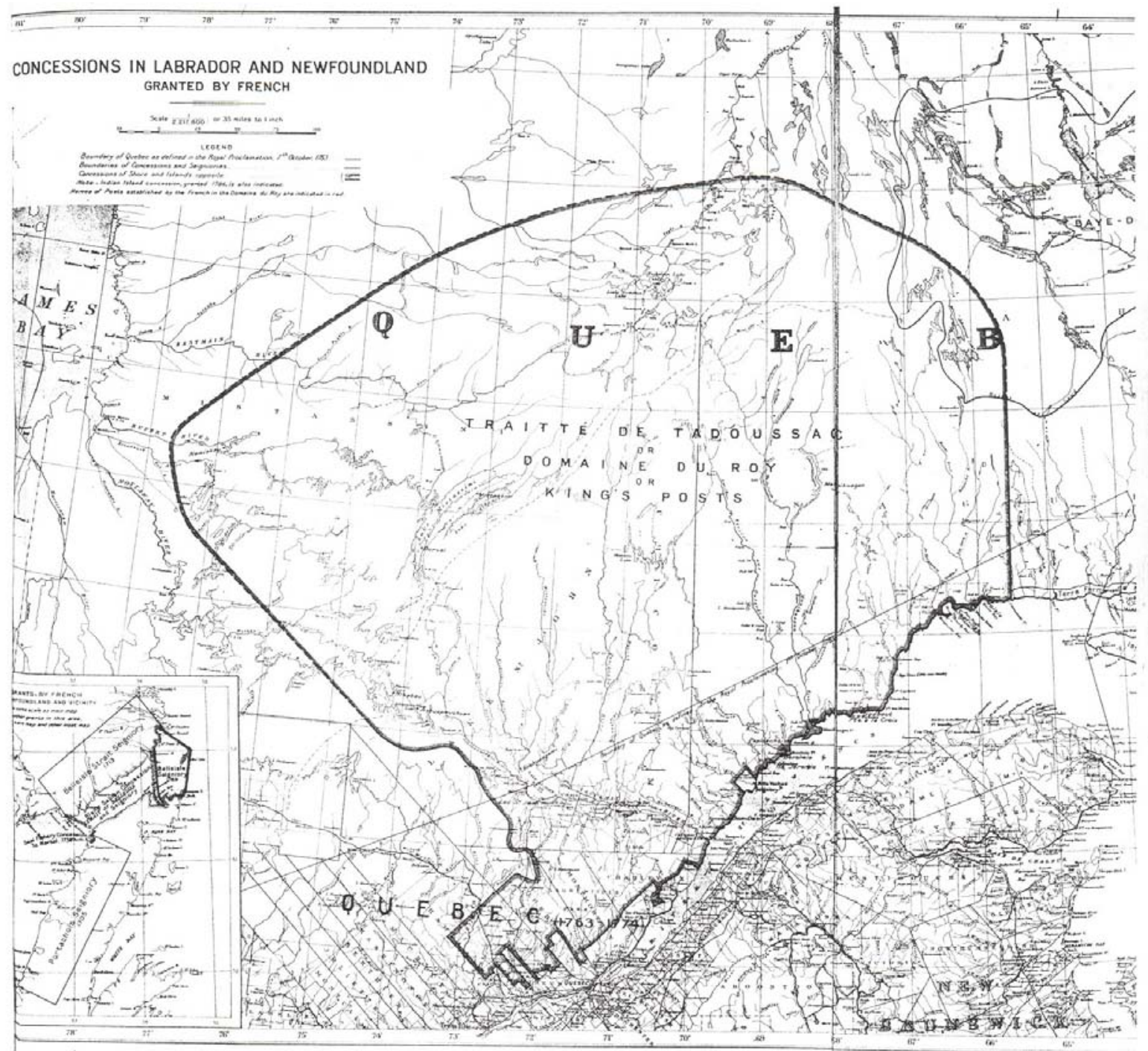

Ajoutons à ces témoignages la conclusion de tous ceux qui ont étudié spécialement la question de ce nom, entre autres les suivants :

a) Les fondateurs de la Société Historique du Saguenay (Mgr J.-Edmond Duchesne, Mgr O.-D. Simard, Mgr Félix-Antoine Savard, Mgr Alphonse
Plourde, Mgr Thomas-Louis Imbeau, Mgr René Bélanger, Mgr Alphonse-Elzéar Tremblay, Mgr LouisJoseph Aubin, les avocats J.-C. Gagné, Percy Martin et Adolphe Routhier, les ingénieurs J.-E.-A. McConville, Édouard Lavoie et Burroughs Pelletier, les notaires Joseph Richard et J.-Omer Lapointe, les abbés Lorenzo Angers, André Laliberté et J.-B. Savard, le 
protonotaire F.-X. Gosselin, l'arpenteur Elzéar Boivin, l'inspecteur d'écoles J.-Édouard Boily, le greffier L.-A. Dussault), auxquels je ne puis m'empêcher d'être associé et dont je suis, avec une équipe dévouée et compétente, le continuateur depuis 30 ans, ce qui me permet d'invoquer à mon crédit 43 années d'étude intensive sur le passé, le présent et les prévisions d'avenir de cette région du Saguenay.

b) Le Comité de Toponymie du Québec, organisme provincial, d'une compétence indiscutable.

c) La Commission du Tourisme, organisme provincial, qui, dans le programme d'une enquête couvrant toute la province, a fait une étude spéciale sur la désignation de la région du Saguenay.

d) L'honorable Lionel Bertrand, alors secrétaire de la Province, et $M$. Robert Prévost, sous-ministre du Tourisme, qui ont participé personnellement à cette enquête et à cette étude.

e) Les fondateurs et les dirigeants de divers organismes opérant dans l'ensemble de la région, tels : La Compagnie Électrique du Saguenay, Saguenay Power et Les Forces motrices du Saguenay, La Compagnie de Téléphone Saguenay-Québec, Saguenay Television Service Company, La Fédération de l'UCC du Saguenay, La Chaîne Coopérative du Saguenay, Le Club des Journalistes du Saguenay, La Société SaintJean-Baptiste régionale du Saguenay...

Cette suite de témoignages établit bien la pérennité du nom traditionnel de la région du Saguenay telle que décrite, depuis l'origine jusqu'à maintenant, avec une autorité et une fermeté qui défient toute contradiction et dissipent tout doute sérieux.

\section{Les autres applications du nom}

Le nom " Saguenay » a été appliqué à d'autres entités géographiques que le territoire auquel il appartient par son origine et son histoire. Voici les cas dignes de mention.

\section{Au cours d'eau}

Les Indiens indiquèrent au découvreur, Jacques Cartier, un premier cours d'eau par où on pouvait pénétrer dans le pays du Saguenay. Pour cette raison Car- tier donna le nom de "rivière du Saguenay » à ce cours d'eau, dont le nom indien était Pitchitaouitchez. Une partie de ce cours d'eau est un fjord, l'autre est une rivière qui est la décharge du lac Saint-Jean. Sur les cartes et dans l'usage courant on désigne généralement les deux sous le nom de « rivière Saguenay ».

Ce nom n’a jamais changé, si ce n'est sur quelques cartes où on donne à la rivière son nom primitif.

Une erreur très commune actuellement est de croire que c'est la rivière qui donne son nom à la région du Saguenay, et conséquemment d'accepter une « région du Saguenay » qui se limiterait au voisinage de cette rivière. La vérité est exactement le contraire. Saguenay est le nom d'un pays, d'un territoire, qu'il décrit même par sa signification (pays « d'où l'eau sort »); il a été appliqué à la rivière par le découvreur (qui n’en connaissait probablement pas le nom) pour indiquer qu'elle était le chemin par où on pouvait se rendre dans le Royaume du Saguenay. Si celui-ci avait été désigné par le nom de la rivière il aurait été appelé « Pitchitaouitchez ». Ici c’est le nom du pays qui a été appliqué au cours d'eau.

\section{Le nom « Saguenay » a été appliqué à d'autres entités géographiques que le territoire auquel il appartient par son origine et son histoire.}

\section{La division électorale}

À la session de 1829 la Législature du Bas-Canada a détaché de l'ancien comté de Northumberland le comté de Montmorency et a donné le nom Saguenay à la partie restante, qui comprenait exactement le territoire historique du Saguenay et en plus le prolongement de la Côte Nord, du golfe Saint-Laurent jusqu'au détroit de Belle-Isle. Ce fut le premier comté de Saguenay.

En 1853, ce comté fut divisé en trois. La partie sud, correspondant au comté actuel de Charlevoix garda le nom «Saguenay » et tout le reste forma les comtés unis de "Chicoutimi et Tadoussac », avec ligne de séparation au méridien $70^{\circ} 15^{\prime}$. Ce fut le deuxième.

En 1858, la Législature remplaça le nom « Saguenay » par celui de "Charlevoix » pour le petit comté créé en 1853 et le substitua à celui de « Tadoussac » 
pour le grand comté s'étendant du méridien $70^{\circ} 15^{\prime}$ au détroit de Belle-Isle. Ce fut le troisième comté de Saguenay. Il contenait la partie est du territoire historique du Saguenay et s'étendait aussi loin en dehors. Il a duré plus de cent ans.

En 1960, cet immense comté fut divisé à la rivière Sainte-Marguerite, un peu à l'ouest de Sept-Iles; la partie est fut appelée « comté de Duplessis » et la partie ouest garda le nom de " comté de Saguenay ». Il aurait été préférable, au point de vue toponymique, de donner à celui-ci un autre nom, pour éviter les inconvénients qui résultent de la désignation de deux territoires (celui du comté et celui de la région) par le même nom.

\section{Au canton}

Un canton situé du côté sud du fjord Saguenay, à son débouché dans le fleuve Saint-Laurent, a été érigé en 1863 et inscrit au terrier sous le nom de « Saguenay ». Il offre peu de terre propre à l'agriculture et de lieux favorables aux établissements, de sorte qu'il occupe peu de place dans l'emploi courant.

\section{L'application des termes « région du Saguenay », ou « Saguenay » au sens de région, à la vallée de la rivière Saguenay est d'origine récente et n'est pas autorisée ni justifiée.}

\section{À deux autres entités}

Une rivière qui se décharge dans le fjord Saguenay à environ 20 milles en amont de Tadoussac s'appelle "Petit-Saguenay ». Nous ne savons pas quand ni comment ce nom lui a été donné.

Une localité sous le nom de "Petit-Saguenay » a été établie sur le cours de cette rivière en 1848. Elle s'appelle encore ainsi.

\section{Un emploi fautif}

L'application des termes "région du Saguenay », ou "Saguenay » au sens de région, à la vallée de la rivière Saguenay est d'origine récente et n'est pas autorisée ni justifiée. Elle est l'effet d'un défaut d'observation ou de connaissance, mais elle a été popularisée avec intensité, de sorte qu'il faut en tenir compte dans l'histoire, même si, comme il faut le souhaiter, elle devrait disparaître bientôt.

Cet emploi est fautif. En effet il introduit dans la toponymie régionale une confusion inextricable, en mettant sur le même plan deux régions du Saguenay: la grande, ainsi désignée depuis toujours, comprenant six régions particulières, et une petite qui est une de ces régions particulières faisant partie de la grande.

Cette confusion devient une vraie Babel quand il s'agit de mentionner ensemble la région du Lac SaintJean et celle de Chicoutimi, que leur situation géographique et leurs relations d'ordre économique, social, religieux et politique associent intimement au point d'obliger à en parler très souvent comme si elles n'en formaient qu'une. Quand, au lieu de dire: « les régions de Chicoutimi et du Lac Saint-Jean », ou même : " la région Chicoutimi-Lac Saint-Jean », on appelle cela "Saguenay-Lac Saint-Jean », on mêle complètement les cartes par la substitution de nom. Le nom "Saguenay » est là hors de sa place; il est appliqué à autre chose que le Saguenay réel.

Normalement c'est un non-sens. Quand on a dit « Saguenay », on a nommé le territoire qui contient la région du Lac Saint-Jean; y ajouter le nom de celle-ci indiquerait qu'on ne connaît pas ce détail élémentaire; c'est comme appeler le pays «Canada-NouveauBrunswick » ou dire « la Mauricie-Shawinigan ».

Cet emploi fait supposer que ce qu'on appelle ainsi "Saguenay » est la région attenante à la rivière Saguenay et non pas la région même du Saguenay, ce qui n’est pas moins déroutant et fautif. Pour éviter l'équivoque, il faudrait joindre au nom "Saguenay » le mot " rivière " afin d'indiquer de quelle région on parle, et dire : "la région de la Rivière Saguenay », comme on emploie le mot « lac » avec le nom SaintJean, pour identifier cette région et ne pas la confondre avec celle de Saint-Jean dans le sud de la province.

Mais alors on crée une autre confusion. Une région de «la Rivière Saguenay » engloberait une partie de celle du Lac Saint-Jean et précisément celle qui forme le comté nommé « Lac-Saint-Jean ». Une ville comme Alma, qui est indiscutablement dans la région du Lac Saint-Jean, serait, de façon aussi indiscutable, dans 
celle de la Rivière Saguenay, puisqu'elle est physiquement à cheval sur cette rivière.

Pour respecter l'intégrité de la région du Lac SaintJean il faudrait limiter la "région de la Rivière Saguenay » à la partie inférieure du cours de la rivière, et il faudrait faire la même chose de l'autre côté pour respecter l'intégrité de la région de la Côte-Nord. D’où l'on voit que l'expression « région de la Rivière Saguenay », tout comme "Saguenay » tout court, pour désigner ce qui est en réalité la région du milieu du cours de la rivière Saguenay est fausse et inadéquate. Cette région mitoyenne doit s'appeler, comme par le passé "région de Chicoutimi », et pour désigner les deux associées c'est l'expression « Chicoutimi-Lac Saint-Jean » qui doit reprendre sa place dans l'emploi.

Observons que dans cet emploi le nom «Chicoutimi » est justifié par le rôle de la ville qui est le centre administratif de la région traversée par le cours inférieur de la rivière et la partie ouest du fjord; il l'est aussi par le sens du mot "Chicoutimi » luimême, qui signifie " jusqu'où c'est profond » et désigne bien la région située à la tête de la navigation.

\section{Le titre de « royaume "}

Le nom de la région du Saguenay est apparu dans l'histoire associé au titre de "royaume » et il y est resté associé jusqu’à maintenant. La valeur historique et publicitaire de ce titre n'affecte aucunement l'authenticité du nom de la région, qui demeure indiscutable; il y a lieu cependant d'en vérifier le bienfondé.

La première mention de ce titre se trouve dans la relation du deuxième voyage de Cartier, à la date du 13 août 1535. La voici dans son contexte : « Et par les deux sauvages que avions pris le premier voyage nous fut dit... que à deux journées du dit cap et île (Anticosti) commençait le royaume du Saguenay, à la terre de devers le nord...»

Jacques Cartier a cru à l'existence, tout au moins à la possibilité d'un royaume dans le territoire du Saguenay. Ce territoire faisant partie de la masse continentale où, plus au sud, les Espagnols avaient trouvé des empires et des civilisations brillantes, il était vraisemblable et tout à fait normal d'en trouver aussi à cette latitude. Les Indiens rencontrés à la côte du
Saint-Laurent n'étaient pas de condition plus primitive que ceux qui habitaient le bord de la mer au Mexique, au Yucatan et au Pérou; on pouvait penser en trouver, comme là-bas, des plus évolués dans l’intérieur des terres.

Le découvreur tâcha de se renseigner le plus possible sur le pays et ramena même en France avec lui ceux qui prétendaient bien connaître le royaume du Saguenay. Plus tard, lui-même et le sieur de Roberval, qui comptait le Saguenay dans le domaine de sa viceroyauté, firent des efforts pour s'y rendre. Et textes et cartes attestent que l'idée d'un royaume dans cette région a persisté pendant au moins une vingtaine d'années. Le royaume tel qu'on l'avait imaginé, avec des châteaux, des rois et des armées, a été ensuite regardé comme n'existant pas, de même que la présence des mines, dont la réalité n'a été constatée qu’à notre époque.

Y avait-il là quelque chose qui aurait pu répondre autrement à l'idée d'un royaume ? Une sorte d'État qu'à défaut de terme mieux approprié il fallait appeler ainsi pour indiquer qu'il existait réellement, comme ceux d'Europe, presque tous des royaumes à cette époque?

Ce qu'on connaît du Saguenay primitif suffit pour attester que les peuples qui y vivaient possédaient une organisation, sans charte écrite mais réelle quand même, adaptée aux conditions matérielles et sociales et faisant de leur pays un ensemble ressemblant à un royaume, bien que moins compliqué et plus démocratique que la plupart. Il y régnait de l’ordre, de la discipline, des lois respectées, une vie économique mieux planifiée que la nôtre actuelle, une sorte de confédération mieux comprise et mieux appliquée que celle que nous nous sommes donnée.

Chaque groupe ou tribu avait ses territoires de chasse déterminés, dont la distribution était réajustée chaque année dans les réunions générales, il avait ses chefs élus et un grand sagamo régional, également élu, pour présider aux délibérations et agir comme arbitre ou juge dans les difficultés entre chefs ou tribus. L'embouchure de la rivière Métabetchouan était le lieu traditionnel des assises plénières; le Père Albanel écrit qu'il y a «vu jusqu'à vingt nations réunies ». Il faut lire la description de ces réunions pour constater combien nos parlements sont loin de présenter un pareil exemple de ponctualité, de tenue et de sérieux. Les problèmes étaient étudiés et solutionnés à l'avance et 
les cas étaient réglés selon l'ordre établi. Dans trois cents ans, en dépit de périodes très difficiles et des complications amenées par la présence des blancs, on n'a pas enregistré un seul cas de guerre entre les tribus du Saguenay, ni de tiraillements, ni de velléités de séparatisme.

Appeler cela « royaume » ne serait pas une hérésie si on savait conserver à ce mot la plénitude de son sens. Les Indiens, qui avaient vu en France un grand chef avec des chefs subalternes dans les provinces, des règlements imposés, des juges pour trancher les conflits, des terres distribuées et des échanges de produits, ont révélé aux blancs que le Saguenay possédait tout cela à sa manière. Les blancs n'ont pas cherché d'autre terme que celui de "royaume » pour qualifier ce pays. Avaient-ils tort ? Bien des pays qu'on appelle "royaume » parce que leur chef a le titre de « roi », dans le passé et encore de nos jours, notamment en Afrique, ne sont pas plus brillants que celui du Saguenay en 1535 et après.

Voilà un premier fait qui permet d'accepter le terme sans offenser la pudeur. Un deuxième fait, d'une plus rigoureuse valeur historique encore, c'est que le Saguenay est entré dans l'histoire avec le titre de " royaume ». Ce fait est indiscutable, que le royaume lui-même soit discutable, légendaire ou réel. La région du Saguenay actuelle étant authentiquement celle qui s'appelait à son origine « le royaume du Saguenay », elle a parfaitement le droit de garder son titre comme son nom.

\section{Dans trois cents ans, en dépit de périodes très difficiles et des complications amenées par la présence des blancs, on n'a pas enregistré un seul cas de guerre entre les tribus du Saguenay, ni de tiraillements, ni de velléités de séparatisme.}

D'autres faits de son histoire et de sa condition nous autorisent à lui conserver ce titre au sens métaphorique. La grande région du Saguenay a été domaine royal pendant plus de cent ans. Elle a été royaume des fourrures, royaume du pin blanc, royaume du tourisme; elle est encore royaume de la ouananiche, royaume des bleuets, du papier, de l'aluminium, de l'électricité; elle peut redevenir royaume du tourisme. Au- tant qui s'ajoute à l'acquis historique, à l'usage quatre fois séculaire et à la situation géographique du Saguenay pour lui donner droit au titre de « royaume ».

\section{Conclusion}

La conclusion de cette étude sur le nom du Saguenay dans l'histoire rejoint celle du Comité de Toponymie du Québec, telle que formulée dans la décision qu’il a rendue à la séance qu'il a tenue le 16 juin 1961. Je ne saurais mieux faire que de citer textuellement cette décision, qui éclaire en même temps tout le problème des désignations régionales dans le Saguenay.

«1. La région géographique naturelle qui comprend les comtés de Saguenay, Chicoutimi, Jonquière-Kénogami, Lac-Saint-Jean, Roberval, doit continuer de s'appeler Saguenay, comme depuis son origine, ou si l'on veut Royaume du Saguenay.

«2. Chacun des secteurs compris dans le Saguenay doit retenir le nom qui lui est consacré. Il n’est donc pas question de faire disparaître le nom de Lac-SaintJean, qui désigne en particulier tout le territoire que baigne le lac; de même pour les noms de Côte-Nord, Chicoutimi, etc.

«3. L'expression Saguenay-Lac-Saint-Jean est inacceptable parce que sa seconde partie est déjà comprise dans la première. C'est également un nom d'une longueur fastidieuse, de même qu'un curieux assemblage d'un toponyme indien avec un nom composé français.

« 4. Il serait préférable qu’il n’y eût pas de comté portant le nom de Saguenay, car le territoire de celuici ne coïncide que partiellement avec la région du même nom, d'où confusion et illogisme.

«Le Comité de Toponymie du Québec recommande donc catégoriquement l'usage du mot Saguenay pour désigner l'ensemble du territoire, à l'intérieur duquel on pourra nommer des secteurs bien délimités et typiques, au moyen des toponymes suivants :

«Lac-Saint-Jean pour la partie que baigne le lac du même nom;

"Côte-Nord pour une partie de la rive du Saint-Laurent qui s'étend à l'est de l'embouchure du Saguenay (fjord); 
«Chicoutimi, qui comprend le comté du même nom et celui de Jonquière-Kénogami;

«Chibougamau, qui englobe la ville et le lac du même nom ainsi que le territoire avoisinant.

« De nouveaux toponymes viendront sans doute s'ajouter aux quatre précédents, à mesure que d'autres parties du Saguenay connaîtront un essor particulier et acquerront des caractéristiques plus marquées. Elles continueront toutefois de participer à cette grande entité géographique qu'est la région du Saguenay. »

En présentant cette décision, le Comité de Toponymie du Québec écrivait qu'après avoir « fait une étude approfondie de la question, en tenant compte rigoureusement de la réalité géographique, des faits historiques et des convenances toponymiques, il est venu à la conclusion très nette que ce nom (Saguenay), qui de tout temps a désigné le même territoire, s’identifie avec la région et doit lui être conservé ».
LE PEUPLE QUI SE SOUVIENT - Heureux le peuple qui n'oublie pas ce que la Providence a fait pour lui, qui consacre des jours de fête publique à la commémoration des grands événements de son histoire; heureux le peuple qui garde le souvenir durable des œuvres de ses ancêtres, qui célèbre ses anniversaires glorieux au pied des autels du Dieu de la patrie; Il sera digne d'estime et de bonheur, il recevra une grande gloire et un nom éternel.

Mgr Antoine Racine Sermon au deuxième centenaire du Séminaire de Québec

\section{Notes et références}

1 Cet article est paru une première fois dans la revue Saguenayensia, vol. 5, n 5-6, sept-oct-nov-déc 1963. Nous le reproduisons avec l'autorisation de la Société historique du Saguenay.

2 Une étude précise de ces cartes pour la période de 1536 à 1600 a été publiée dans Saguenayensia, juillet-août 1963. 